MATEC Web of Conferences 11,03003 (2014)

DOI: $10.1051 /$ matecconf / 20141103003

(C) Owned by the authors, published by EDP Sciences, 2014

\title{
Identification of formations of soil and subsoil using finite elements modeling
}

\author{
Latifa OUADIF ${ }^{1}$, Lahcen BAHI ${ }^{1}$, Khadija BABA ${ }^{1}$ \\ ${ }^{1}$ Laboratoire L3GIE, Ecole Mohammadia d’Ingénieurs, Université Med V Agdal, Rabat, Maroc.
}

\begin{abstract}
The Soil and subsoil survey are performed by direct and indirect methods. The direct methods are the investigations of field and laboratory while indirect methods are the geophysical methods. These allow, from one or more physical properties, to find out the underground's structure nondestructively.

Geophysical methods for characterizing subsurface have long been used, in geotechnical, hydrogeological and environmental studies. For our study, we used the electrical resistivity's method. 52 geoelectric soundings VES was performed with a mesh of $500 \mathrm{~m}$ and electrodes distance between $1000 \mathrm{~m}$ and $3000 \mathrm{~m}$. The interpretation of these VES allows the determination of the vertical succession of formations in place. In order to have a lateral facies variation, we used geostatistical methods for having adequate data that can be used in the input file of inversion's code Res2Dinv.

The 2D images of the subsoil obtained confirm and complement the results of the correlations of oil drilling that are in this region. Thus, the study area also has a horst and graben structure. This study also allowed us to locate the sandstone formations that extend in two places along a North-South direction.
\end{abstract}

\section{Introduction :}

Le site de Outita se situe dans la province de Sidi Kacem. Il est bordé au nord par la route secondaire $\mathrm{n}^{\circ}$ 229 reliant la localité de Dar Bel Amri et la ville de Sidi Kacem (Fig.1). Le domaine est situé dans la partie orientale de la plaine du Gharb, au pied des collines de Koudiat Essma et de Jbel Outita. Cette région fait partie du bassin du Gharb. Ce dernier est une dépression limitée au Nord par le Prérif, à l'Est par les rides prérifaines, au Sud par la meseta marocaine et à l'Ouest par l'Océan Atlantique. Le bassin du Gharb a connu des affaissements durant certaines périodes qui s'accentuent au Pliocène [1, $2,3]$. La complexité structurale de la bordure orientale du bassin du Gharb rend difficile la détermination et le suivi des formations constituées par des dépôts perméables susceptibles de correspondre à des niveaux aquifères [4, $5,6]$.

La localisation et la délimitation des formations gréseuses sont confrontées aux difficultés posées par le manque de données, ce qui nécessite des études synthétiques impliquant la géologie locale, l'étude des forages implantés dans la zone et une campagne géophysique basée sur la méthode des résistivités électriques.

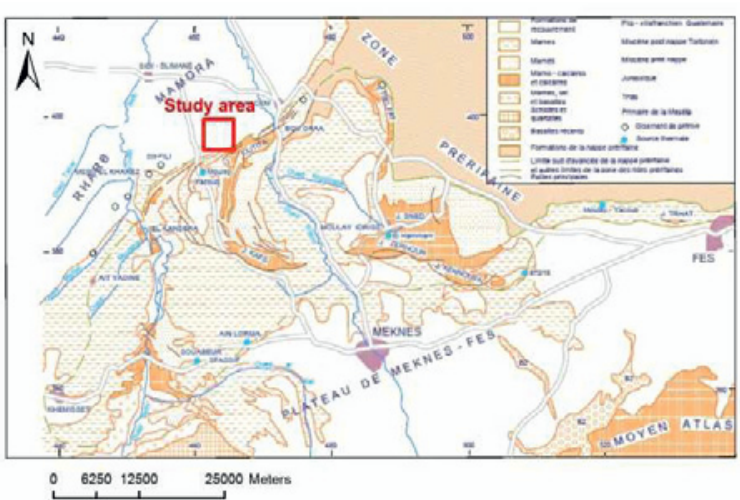

Fig. 1. Situation géographique et géologique de la région d'étude [7]

\section{Matériel et Méthodes :}

L'objectif de l'étude est de localiser et délimiter les formations gréseuses et d'identifier les caractéristiques structurale de la subsurface. Cette approche a nécessité l'interprétation de 52 sondages électriques dont la longueur de la ligne $\mathrm{AB}$ varie de $1000 \mathrm{~m}$ à $3000 \mathrm{~m}$, effectués dans la région de Sidi Kacem d'une part, et l'étude des forages implantés dans la région qui ont fournis une base de données sur les faciès 
pétrographiques des dépôts jurassiques et néogènes de la limite du bassin avec les rides prérifaines d'autre part (Fig 2).

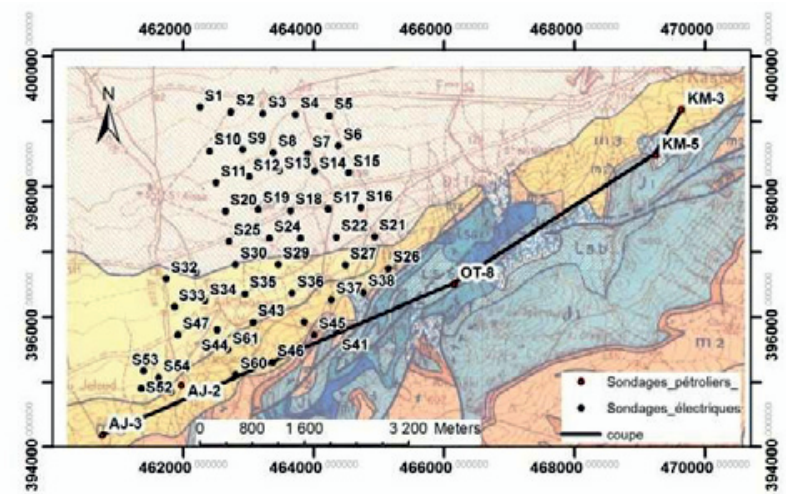

Fig. 2. Situation des sondages électriques et des forages pétroliers.

Dû à son faible coût, la méthode de résistivités électriques est la plus utilisée en génie civil, en géologie appliquée et en études environnementales. Elle permet d'identifier à partir de la surface les structures qui présentent des contrastes de résistivité et aussi de les localiser [8,9]. Elle consiste à réaliser des sondages électriques permettant de déterminer en plusieurs points la succession verticale d'horizons de résistivités différentes. Or l'interprétation des sondages verticaux donne la succession verticale des formations à l'aplomb de chaque sondage [10]. Pour délimiter la formation gréseuse, il faut avoir des images $2 \mathrm{D}$ du sous sol. Ces images sont réalisées par tomographie électrique, appelée aussi panneau électrique, qui consiste en la mesure des résistivités apparentes du sous sol selon un dispositif rectiligne d'électrodes plantées dans le sol. Il est nécessaire, alors, de transformer les mesures de résistivités apparentes obtenues par les sondages électriques en données pouvant être obtenues par la technique de tomographie électrique (TRE). Pour estimer les résistivités apparentes aux points de TRE, on a eu recours au krigeage ordinaire où les résistivités apparentes sont linéairement estimées au moyen de l'équation :

$\log \left(\rho_{a p p_{i}}^{*}\right)=\sum_{\mathrm{i}=1}^{\mathrm{N}} \gamma_{\mathrm{i}} \log \left(\rho_{\mathrm{app}_{\mathrm{i}}}\right)$ avec $\sum_{\mathrm{i}=1}^{\mathrm{N}} \gamma_{\mathrm{i}}=1$

L'inversion de ces données est faite à l'aide du code à éléments finis RES2DINV qui utilise la méthode de lissage par moindres carrés appelée méthode d'inversion de norme L2 [11,12]. Le modèle 2-D utilisée par ce programme divise le sous-sol en un certain nombre de blocs rectangulaires. Le procédé d'optimisation tente essentiellement à réduire la différence entre les valeurs de résistivité apparente calculées et mesurées par l'ajustement de la résistivité des blocs du modèle. La mesure de cette différence est donnée par la racine quadratique moyenne de l'erreur notée RMS.

\section{Résultats et discussions}

Les corrélations des forages pétroliers (Fig. 3) montrent que les terrains jurassiques et néogènes sont affectés à la fois par des failles normales qui provoquent un effondrement de part et d'autre d'une zone haute formée par des dépôts jurassiques (forages OT8 et KM5) avec épaississement des marnes du Miocène, et des failles inverses qui délimitent des écailles tectoniques. Ces failles inverses sont dues principalement à une traction provoquée par l'avancée de la nappe prérifaine dans le bassin Néogène du Gharb. Ceci confirme la complexité de cette zone qui est affectée de failles normales et inverses délimitant des horsts et des grabens.

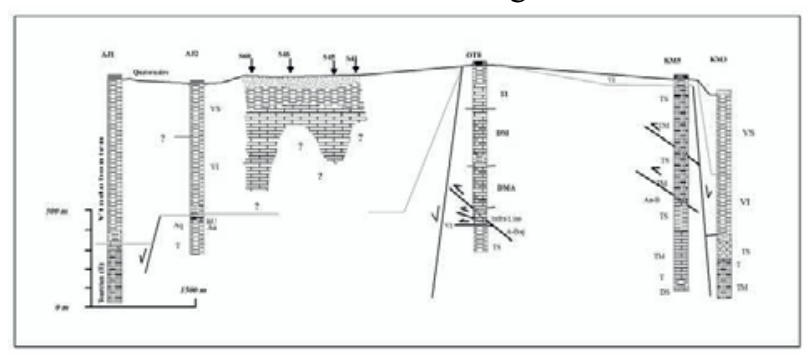

Fig. 3. Coupe géologique et géoélectrique de direction NE-SW [10].

Les cartes de krigeage ont été faites sur une grille à maille carrée de coté $60 \mathrm{~m}$. Les données issues de ces cartes sont organisées dans des fichiers compatibles avec ceux qui sont exploitables par le code à éléments finis RES2DINV. Six profils de direction Ouest-Est sont établis et d'après les résultats obtenus deux autres profils de direction Nord-Sud sont ajoutés pour déterminer l'étendue latérale des formations gréseuses caractérisées par de grandes résistivités (Fig. 4).

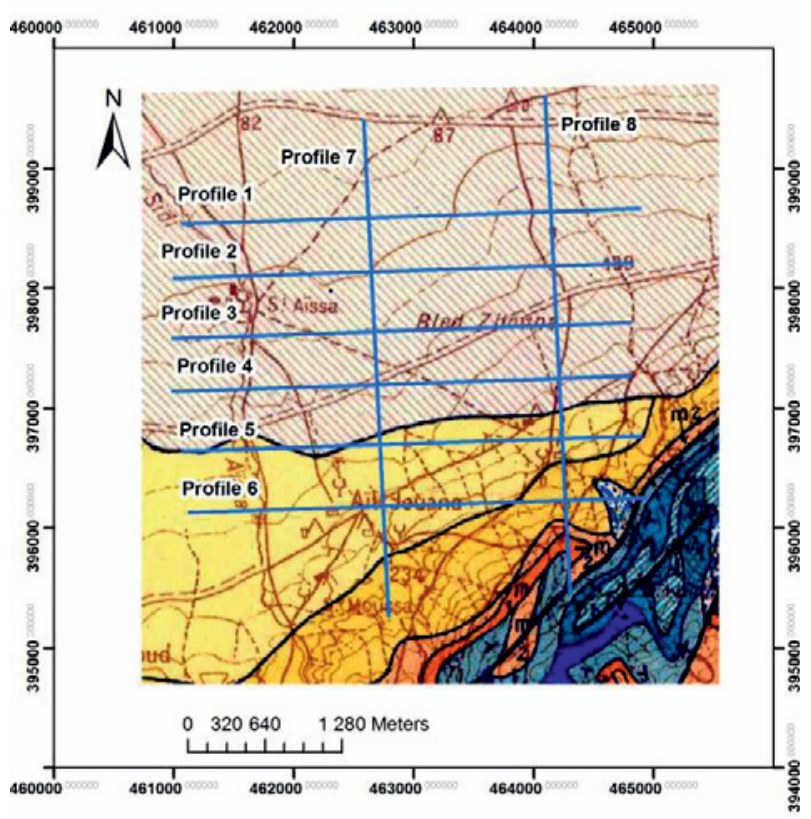

Fig. 4. Situation des profils TRE

Les profils géoélectriques (profil 1 à 6) orientés Ouest-Est (Fig. 5) montrent des valeurs de résistivité qui 
varient latéralement et en profondeur. Nous distinguons trois niveaux selon les valeurs de résistivité obtenues. Dans la partie supérieure, il existe un niveau de haute résistivité. Ce niveau correspond à des conglomérats qui affleurent par endroits. Le deuxième niveau présente des résistivités très faibles. Il correspond aux dépôts marneux du Néogène. Enfin, en profondeur, il y a un troisième niveau caractérisé par des résistivités élevées. Il correspond aux formations gréseuses. Ces formations sont situées à des profondeurs de 300 à $400 \mathrm{~m}$ à l'aplomb des coordonnées Lambert $\mathrm{x}=462587 \mathrm{~m}$ et $\mathrm{x}=464087 \mathrm{~m}$, pour déterminer leur extension, deux profils orientés Nord-Sud sont établis. Le profil 7 (fig. 6), correspondant à $\mathrm{x}=462587 \mathrm{~m}$, montre que la couche des grès s'étend de $\mathrm{y}=398130 \mathrm{~m}$ à $397010 \mathrm{~m}$ alors que pour le profil 8 correspondant à $\mathrm{x}=464087 \mathrm{~m}$, elle s'étend de $\mathrm{y}=$ 397 730m jusqu'à la frontière sud de la zone.

Les profils orientés Ouest-Est montrent une structure en horst et graben avec un épaississement des marnes du Néogène au centre de la zone d'étude. Ceci confirme et complète les résultats obtenus par corrélation des sondages pétroliers.

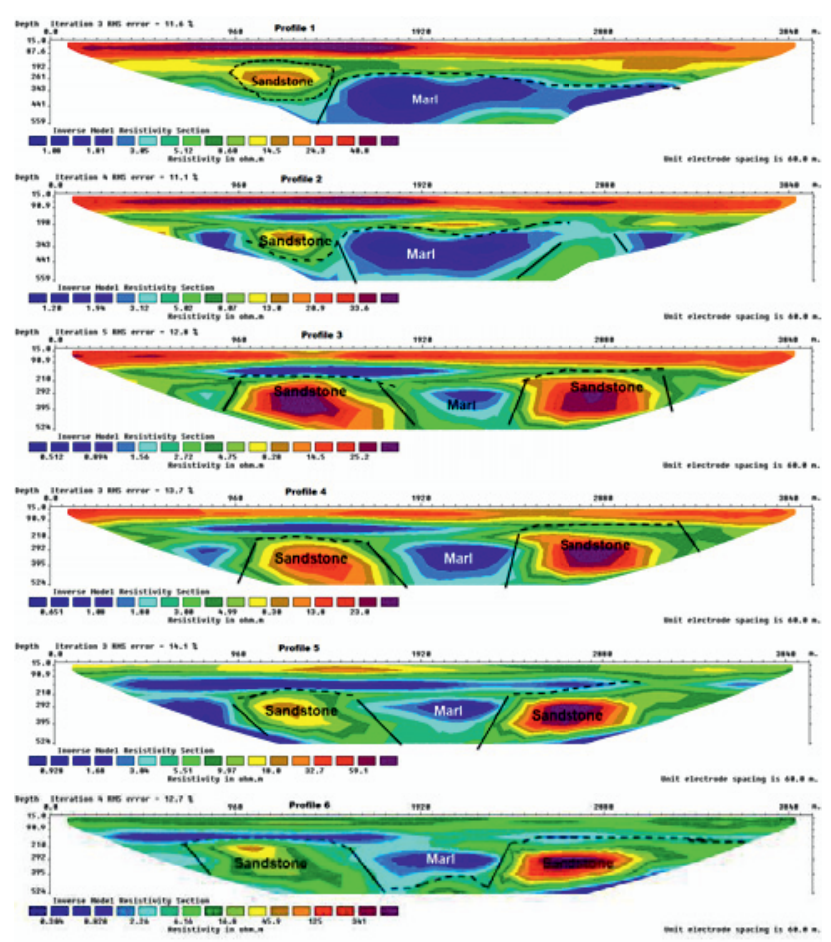

Fig. 5. Modèles d'inversion le long de la direction Ouest-Est.

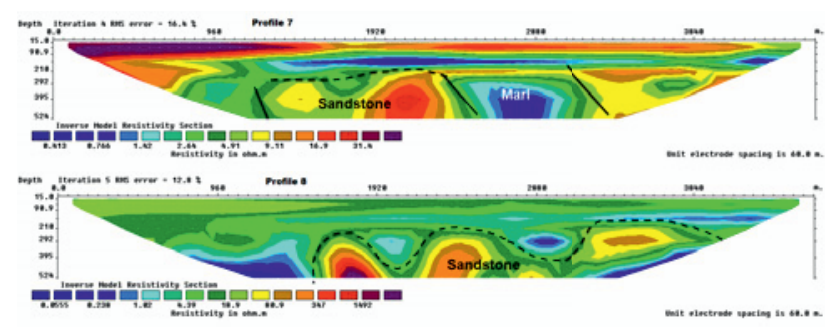

Fig. 6. Modèles d'inversion le long de la direction Nord-Sud.

\section{Conclusion :}

Le bassin du Gharb a fait l'objet de plusieurs études géologique, sédimentologique et géophysique, cependant la limite Est du bassin reste encore méconnue. Les corrélations des forages pétroliers effectués dans la région de Sidi Kacem montrent des failles inverses affectant le jurassique et le Néogène, dues à une traction provoquée par l'avancée de la nappe prérifaine. La méthode des résistivités électriques a montré la complexité géologique de cette bordure. Les images 2D de la TRE montrent une structure en horst et graben. Ceci confirme et complète les résultats des corrélations des forages pétroliers.

Les profils de tomographie électrique ont permis de localiser les formations gréseuses qui sont atteintes à des profondeurs de $300 \mathrm{~m}$ et de déterminer leur étendue selon une direction Nord-Sud. Ces formations sont susceptibles de constituer des aquifères et résoudre le problème d'eau potable et d'irrigation pour la population rurale de cette région.

\section{Références :}

[1] Litto W. Apport de la sismique réflexion et des diagraphies à l'étude de l'évolution géodynamique d'un bassin néogène à potentiel pétrolier : exemple de la marge nord du bassin du Gharb (Avant pays rifain, Maroc). Thèse de Doctorat, Fac. Sci. Rabat, Maroc, 203p 2001.

[2] Cirac P. 1985. Le bassin sud-rifain occidental au Néogène supérieur. Evolution de la dynamique sédimentaire et de la pléogéographie au cours d'une phase de comblement. Thèse d'état, Univ. De Bordeaux I, 3 tomes.

[3] Feinberg H. 1986. Les séries tertiaires des zones externes du Rif (Maroc): Notes et mémoires du Service Géologique, Maroc, 315, $192 \mathrm{p}$.

[4] Akhssas A. 2002. Contribution à l'étude des niveaux sableux à hydrocarbures du Miocène post-nappe du bassin du Gharb (Maroc). Application de l'analyse multidimensionnelle. Thèse d'état, Univ. Mohammed VAgdal.

[5] Boutakiout M. 1988. Les foraminifères jurassiques des rides sud rifaines et des régions voisines (Maroc). Thèse d'état, Univ. Lyon, 285 p.

[6] Kili, M., El Mansouri B., Chao J., Ait Fora A. De nouveaux éléments structuraux du complexe aquifère profond du bassin du Rharb (Maroc) : implications hydrogéologiques, C. R. Géoscience 338 (2006) 1194 1202.

[7] M. Combe, La Zone prérifaine et les Rides prérifaines, Ressources en Eau du Maroc, Tome 1, Editions Du Service Geologique Du Maroc, Rabat, 1971.

[8] Chouteau T., (2001), Les méthodes électriques, Notes de cours, Publications Ecole Polytechnique, Montréal

[9] Telford W.M, Geldart L.P., Sheriff R.E. and Keys D.A., Prospection géophysique, Tome 3, La Barbannerie, 1989.

[10] Ouadif, L., Bahi, L., Akhssas, A., Baba, K., Menzhi, M., Contribution of Geophysics for the Determination of Aquifers with a Case Study, 
International Journal of Geosciences, Vol. 3 No.1, 2012, pp. 117-125. doi: 10.4236/ijg.2012.31014

[11] deGroot-Hedlin, C. and Constable, S., 1990, Occam's inversion to generate smooth, two-dimensional models form magnetotelluric data. Geophysics, 55, 16131624.

[12] Loke, M. H., and R. D. Barker, 1996, Rapid least-square inversion of apparent Resistivity pseudosection by a quasi-Newton method: Geophysical Prospecting, 44, 131-152. 